\title{
Genome Sequence Resource of Burkholderia glumae UAPB13
}

\author{
Juanita Gil, Laura Ortega, J. Alejandro Rojas, ${ }^{\dagger}\left(\mathbb{D}\right.$ and Clemencia M. Rojas ${ }^{\dagger}$ \\ Department of Entomology and Plant Pathology, University of Arkansas, Fayetteville, AR 72701
}

\section{Genome Announcement}

Burkholderia glumae causes bacterial panicle blight (BPB) in rice, a disease that has significantly affected rice production worldwide (Cui et al. 2016; Ham et al. 2011; Ortega and Rojas 2021; Zhou 2019). Here, we report the genomic sequence of $B$. glumae strain UAPB13 isolated from fields in Arkansas. The assembled genome consists of 123 scaffolds totaling $6,504,483$ bp representing two chromosomes and two plasmids. The genomic complexity of $B$. glumae warrants the sequencing of additional strains. This additional genomic sequence will enable us to further understand this pathogen and the disease it causes.

B. glumae can infect the plant at all developmental stages, causing various symptoms including seed and seedling rot, lesions in leaves, and discoloration in panicles. Infections in panicles at the flowering stage are directly related to yield losses because the bacterium can interfere with fertilization and, as a result, hinder grain development (Fory et al. 2014). The virulence of $B$. glumae has been mainly associated with the toxin toxoflavin (liyama et al. 1995; Karki et al. 2012; Nandakumar et al. 2009). Additional virulence factors have been identified but remain to be fully characterized (Ortega et al. 2020). So far, 21 strains of $B$. glumae have been sequenced (Cui et al. 2021; Fory et al. 2014; Francis et al. 2013; Hussain et al. 2020; Lim et al. 2009), and comparative analyses of these genomes have revealed enormous genomic complexity. Members of this genus often present two chromosomes, and strains present rearrangements often associated with the virulence (Fory et al. 2014; Francis et al. 2013; Lee et al. 2016). Thus, increasing the number of genomes available for this pathogen will enable us to understand more about the pathogen and how it causes disease in rice.

Burkholderia glumae strain UAPB13, initially isolated from rice (Oryza sativa L.) variety Wells in Arkansas County in 2014 (Mulaw et al. 2018), is a virulent strain used in previous studies (Ortega et al. 2020). To obtain the B. glumae UAPB13 genomic sequence, a single colony of $B$. glumae UAPB13 was grown on King's B (KB) agar, transferred to $3 \mathrm{ml}$ of KB broth, and grown for $18 \mathrm{~h}$ at $28^{\circ} \mathrm{C}$. Genomic DNA was extracted using the GeneJET Genomic DNA Purification kit (Thermo Fisher Scientific, Waltham, MA) and quantified using a Qubit 4 fluorometer (Thermo Fisher Scientific). Genomic DNA was sequenced by the Microbial Genome Sequencing Center (MiGS Center, Pittsburgh, PA) using an Illumina tagmentation library approach on a NextSeq 550 instrument with 150-bp paired-end reads (Baym et al. 2015). A total of $7,020,630$ paired-end reads (corresponding to $1,685,160,720$ bp and an estimated

†Corresponding authors: J. A. Rojas; jarojas@uark.edu, and C. M. Rojas; cr022@uark.edu

Data availability: The genome reported here was deposited at NCBI under the Bioproject PRJNA702519 and Biosample accession SAMN17969348.

e-Xtra: A supplementary figure is published online.

The author(s) declare no conflict of interest.

Accepted for publication 4 October 2021.

\section{Funding}

Funding was provided by the University of Arkansas Chancellor's

Commercialization Fund.

\section{Keywords}

bacterial panicle blight of rice, Burkholderia glumae, genome sequence 
Table 1. Genome summary statistics of Burkholderia glumae UAPB13

\begin{tabular}{lc} 
Genomic feature & Result \\
Size $(\mathrm{bp})$ & $6,504,483 \mathrm{bp}$ \\
GC content $(\%)$ & 68.43 \\
Contigs & 146 \\
Contigs $(>200 \mathrm{bp})$ & 123 \\
N50 & $164,212 \mathrm{bp}$ \\
L50 & 10 \\
CDS & 5,557 \\
rRNA & 4 \\
tRNA & 71 \\
\hline
\end{tabular}

a $\mathrm{N} 50=$ contig length that produces $50 \%$ bases of the assembly.

${ }^{b} \mathrm{~L} 50=$ minimum number of contigs that produce $50 \%$ bases of the assembly.

coverage of $88 \times$ ) were obtained, and initial processing consisted of sequence trimming with Trimmomatic v0.39 (Bolger et al. 2014). The genome was assembled using Unicycler v0.4.8 (Wick et al. 2017) with default parameters. Unicycler uses SPAdes v3.14.0 (Bankevich et al. 2012) to correct reads, optimize k-mer selection, and assemble the first draft of the genome. The generated assembly was polished with Pilon v1.23 (Walker et al. 2014), resulting in 298 contigs. A second scaffolding approach was taken using SSPACE v3.0 (Boetzer et al. 2011), resulting in a $6.5-\mathrm{Mb}$ assembly containing 146 scaffolds. Prior to annotation, contigs/scaffolds less than $200 \mathrm{bp}$ were removed, and the assembly was annotated using Prokka v1.14.5 (Seemann 2014). After removing contaminants and contigs less than $200 \mathrm{bp}$, the assembly consisted of 123 scaffolds. The final version of $B$. glumae UAPB13 was aligned to genome B. glumae 257sh-1 (GCA_009931375.1), and scaffolds were mapped to the two chromosomes (3.5 and 2.7 Mb) and two plasmids (212 kb and $174 \mathrm{~kb})$.

The final genome assembly of $B$. glumae UAPB13 was $6.51 \mathrm{Mb}$ with an N50 of 164,212 bp and average GC content of $68.43 \%$ (Table 1). This genome has 5,557 proteincoding sequences (CDS), 4 ribosomal RNA (rRNA) genes, and 71 transfer RNA (tRNA) genes. Genome completeness was assessed with BUSCO v4.0.6 (Benchmarking Universal Single-Copy Orthologs; Seppey et al. 2019) using the bacteria_odb10 database. The resulting completeness indicated that $100 \%$ of the genes in the database were recovered, identifying 124 complete and single-copy BUSCOs.

The genome was evaluated and compared with other available genomes for B. glumae and $B$. gladioli strains using PyANI (Pritchard et al. 2016), resulting in a similarity ranging from 99.4 to $99.9 \%$ based on average nucleotide identity between all $B$. glumae strains (Supplementary Fig. S1). In silico analysis of the genome using BLAST (Altschul et al. 1990) indicated the presence of the toxoflavin operon, and further analysis using antiSMASH (Blin et al. 2019) revealed the presence of biosynthetic genes for the secondary metabolite bactobolin. This metabolite has been associated with cytotoxic activity against other bacteria and eukaryotes (Greenberg et al. 2020). The genome of the UAPB13 strain will provide a resource for understanding BPB in Arkansas and other rice-producing regions in the United States and abroad.

\section{Literature Cited}

Altschul, S. F., Gish, W., Miller, W., Myers, E. W., and Lipman, D. J. 1990. Basic local alignment search tool. J. Mol. Biol. 215:403-410.

Bankevich, A., Nurk, S., Antipov, D., Gurevich, A. A., et al. 2012. SPAdes: A new genome assembly algorithm and its applications to single-cell sequencing. J. Comput. Biol. 19:455-477.

Baym, M., Kryazhimskiy, S., Lieberman, T. D., Chung, H., et al. 2015. Inexpensive multiplexed library preparation for megabase-sized genomes. PLoS One 10:e0128036.

Blin, K., Shaw, S., Steinke, K., Villebro, R., et al. 2019. antiSMASH 5.0: Updates to the secondary metabolite genome mining pipeline. Nucleic Acids Res. 47: W81-W87.

Boetzer, M., Henkel, C. V., Jansen, H. J., Butler, D., and Pirovano, W. 2011. Scaffolding pre-assembled contigs using SSPACE. Bioinformatics 27:578-579.

Bolger, A. M., Lohse, M., and Usadel, B. 2014. Trimmomatic: A flexible trimmer for Illumina sequence data. Bioinformatics 30:2114-2120.
Cui, Z., Wang, S., Kakar, K. U., Xie, G., et al. 2021. Genome sequence and adaptation analysis of the human and rice pathogenic strain Burkholderia glumae AU6208. Pathogens 10:87.

Cui, Z., Zhu, B., Xie, G., Li, B., and Huang, S. W. 2016. Research status and prospect of Burkholderia glumae, the pathogen causing bacterial panicle blight. Rice Sci. 22:111-118

Fory, P. A., Triplett, L., Ballen, C., Abello, J. F., et al. 2014. Comparative analysis of two emerging rice seed bacterial pathogens. Phytopathology 104:436-444.

Francis, F., Kim, J., Ramaraj, T., Farmer, A., et al. 2013. Comparative genomic analysis of two Burkholderia glumae strains from different geographic origins reveals a high degree of plasticity in genome structure associated with genomic islands. Mol. Genetics Genom. 288:195-203.

Greenberg, E. P., Chandler, J. R., and Seyedsayamdost, M. R. 2020. The chemistry and biology of bactobolin: A 10-year collaboration with natural product chemist extraordinaire Jon Clardy. J. Nat. Products 83:738-743. 
Ham, J. H., Melanson, R. A., and Rush, M. C. 2011. Burkholderia glumae: Next major pathogen of rice? Mol. Plant Pathol. 12:329-339.

Hussain, A., Shahbaz, M., Tariq, M., Ibrahim, M., et al. 2020. Genome re-sequence and analysis of Burkholderia glumae strain AU6208 and evidence of toxoflavin: A potential bacterial toxin. Comput. Biol. Chem. 86:107245.

liyama, K., Furuya, N., Takanami, Y., and Noraki, M. 1995. A role of phytotoxin in virulence of Pseudomonas glumae Kurita et Tabeti. Jpn. J. Phytopathol. 61: 470-476.

Karki, H. S., Shrestha, B. K., Han, J. W., Groth, D. E., et al. 2012. Diversities in virulence, antifungal activity, pigmentation and DNA fingerprint among strains of Burkholderia glumae. PLoS One 7:e45376.

Lee, H. H., Park, J., Kim, J., Park, I., and Seo, Y. S. 2016. Understanding the direction of evolution in Burkholderia glumae through comparative genomics. Curr. Genet. 62:115-123.

Lim, J., Lee, T. H., Nahm, B. H., Do Choi, Y., et al. 2009. Complete genome sequence of Burkholderia glumae BGR1. J. Bacteriol. 191:3758-3759.

Mulaw, T., Wamishe, Y., and Jia, Y. 2018. Characterization and in plant detection of bacteria that cause bacterial panicle blight of rice. Res. J. Plant Pathol. 1: 1-7.

Nandakumar, R., Shahjahan, A. K. M., Yuan, X. L., Groth, D. E., et al. 2009. Burkholderia glumae and B. gladioli cause bacterial panicle blight in rice in the Southern United States. Plant Dis. 93:896-905.
Ortega, L., and Rojas, C. 2021. Bacterial panicle blight and Burkholderia glumae: from pathogen biology to disease control. Phytopathology 111:772-778.

Ortega, L., Walker, K. A., Patrick, C., Wamishe, Y., et al. 2020. Harnessing Pseudomonas protegens to control bacterial panicle blight of rice. Phytopathology 110:1657-1667.

Pritchard, L., Glover, R. H., Humphris, S., Elphinstone, J. G., and Toth, I. K. 2016. Genomics and taxonomy in diagnostics for food security: Soft-rotting enterobacterial plant pathogens. Anal. Methods 8:12-24.

Seemann, T. 2014. Prokka: Rapid prokaryotic genome annotation. Bioinformatics 30:2068-2069

Seppey, M., Manni, M., and Zdobnov, E. M. 2019. BUSCO: Assessing genome assembly and annotation completeness. Pages 227-245 in: Gene Prediction: Methods and Protocols. Kollmar M., ed. Springer, New York, NY.

Walker, B. J., Abeel, T., Shea, T., Priest, M., et al. 2014. Pilon: An integrated tool for comprehensive microbial variant detection and genome assembly improvement. PLoS One 9:e112963.

Wick, R. R., Judd, L. M., Gorrie, C. L., and Holt, K. E. 2017. Unicycler: Resolving bacterial genome assemblies from short and long sequencing reads. PLOS Comput. Biol. 13:e1005595.

Zhou, X. G. 2019. Sustainable strategies for managing bacterial panicle blight of rice. In: Protecting Rice Grains in the Post-Genomics Era. Jia Y., ed. IntechOpen, London, U.K. 